\title{
EFECTIVIDAD DEL SISTEMA DE BOMBEO CON ARIETE HIDRÁULICO EN LA ZONA RURAL DE LA PROVINCIA DE SAN PABLO- CAJAMARCA
}

\section{EFFECTIVENESS OF THE PUMPINGSYSTEM WITH HYDRAULIC RAMMING IN THE RURAL ZONE OF THE PROVINCE OF SAN PABLO - CAJAMARCA}

iD Denis Javier Aranguri Cayetano ${ }^{1}$

${ }^{1}$ Universidad Nacional del Santa, Ancash, Perú.

Correspondencia:

Dr. Denis Javier Aranguri Cayetano

daranguri@unitru.edu.pe

\section{RESUMEN}

Esta investigación determinó la efectividad del sistema de bombeo con ariete hidráulico en la zona rural de la provincia de San Pablo-Cajamarca, analizando los parámetros hidráulicos de una bomba de agua, de acuerdo al área en estudio y la configuración adecuada para su instalación, la eficiencia del sistema, así como el grado de satisfacción de los habitantes rurales. Se recolectó, la información mediante entrevistas, observación directa de campo, técnicas de medición de parámetros hidráulicos, y para determinar el grado de satisfacción, se utilizó la escala Likert. La demanda total del recurso hídrico fue $8.072 \mathrm{l} / \mathrm{min}$; lo cual se considera demanda doméstica, ganado y riego de pasturas, mientras que la oferta, 15 a $25 \mathrm{l} / \mathrm{s}$ de agua. Los parámetros de diseño y operación del sistema de bombeo se rigen para un cuerpo de bomba de 1", siendo el sistema en paralelo, proporcionando $8.9 \mathrm{l} / \mathrm{min}$, mostrando una eficiencia total del sistema de $55.23 \%$. El nivel de satisfacción fue positivo en los aspectos técnicos, económicos y ambiental; así como, al uso y aplicación del sistema de bombeo con ariete hidráulico.

Palabras clave: efectividad, bombeo, demanda, ariete hidráulico.

\section{ABSTRACT}

This research work seeks to determine the effectiveness of the Hydraulic Water Pumping System in the rural area of San Pablo - Cajamarca Province, analyzing the hydraulic parameters of a water pump, according to the area under study and the appropriate configuration for its installation, the efficiency of the system as well as the degree of satisfaction of the rural inhabitants. For the collection of information, interviews, direct observation of the field, hydraulic parameters measurement techniques, and to determine the degree of satisfaction, the Likert scale were used. The total demand for water resources is $8,072 \mathrm{l} / \mathrm{min}$; which considers domestic demand, livestock and irrigation of pastures, while the supply is 15 to $25 \mathrm{l} / \mathrm{s}$ of water. The parameters of design and operation of the pumping system is governed for a 
pump body of 1 ", being the system in parallel; providing $8.9 \mathrm{l} / \mathrm{min}$ where the total efficiency of the system is $55.23 \%$. The level of satisfaction of the inhabitants is positive, from the technical, economic and environmental aspects to the use and application of the pumping system with hydraulic ram.

Key words: effectiveness, pumping, hydraulic ram

\section{INTRODUCCIÓN}

La falta de acceso a una fuente de agua; así como, la carencia de sistemas de saneamiento no sólo conlleva a una degradación sustancial del nivel de vida, sino que, trae consigo una cantidad de consecuencias directas como por ejemplo: limitación en el uso del recurso hídrico en actividades domésticas (se requiere para la eliminación de desperdicios en forma satisfactoria: para el baño, limpieza y aseo personal, lavado de ropa, lavado de trastos), suministro de agua para la agricultura, incluyendo ganado e irrigación, entre otras. Por el contrario, si la zona en mención tuviera acceso a energía eléctrica, el consumo excesivo de energía en un sistema de bombeo, se vería reflejado en la facturación, con los consiguientes efectos negativos en la economía del poblador rural (Ortega \& Gonzáles, 2013).

Entre otras, esta es una causa que obliga a tomar en cuenta la posibilidad de utilizar y/o aplicar otras fuentes de energía renovables, como la hidráulica, eólica, solar etc., en proyectos que benefician al hombre (JICA, 2012; Mayorga \& Galarza, 2013).

En la actualidad, existen sistemas de alta eficiencia para transformar la energía hidráulica en energía eléctrica y mecánica (Nwosu \& Madueme, 2013).

En relación a la bomba de ariete, a diferencia de las bombas convencionales, es hidráulica cíclica, la cual utiliza la energía cinética de un golpe de ariete en un fluido, para subir una parte de éste a un nivel superior, sin emplear otra fuente de energía externa. Por sus grandes ventajas, está siendo retomada en países como Cuba, Chile y Bolivia, donde incluso se han creado centrales de bombeo donde se usa el ariete para abastecer de agua a pequeñas ciudades, incluyendo universidades y organizaciones que realizan mejoras a este sistema.

En el Perú, el grupo de apoyo al sector rural (Grupo PUCP), es una unidad operativa del Departamento de Ingeniería de la Pontificia Universidad Católica del Perú (PUCP). Desde el año 1992 viene realizando proyectos de desarrollo a favor de comunidades campesinas, relacionados con la investigación científica aplicada, transferencia e innovación tecnológica, difusión y promoción de tecnologías apropiadas y actividades de conservación del medio ambiente; cuyo objetivo es mejorar la calidad de vida de la población rural en los ámbitos de energía, agua, agricultura y vivienda.

Según Arapa (2016), la tecnología de bomba de ariete no es muy conocida; así como, tampoco ha gozado de amplia difusión en el norte del país. Por consiguiente, la realización de esta investigación, evidenciará la disponibilidad de un desarrollo institucional como apoyo de un importante elemento de promoción y soporte a la difusión de estos sistemas de bombeo. Por lo tanto, este sistema puede ser adaptado fácilmente a las condiciones geomorfológicas e hidrológicas en algunas zonas rurales de la provincia de San Pablo de la región de Cajamarca, al permitir el bombeo de las partes bajas de los ríos u otros, hacia las zonas altas, con el fin de satisfacer la provisión de agua y mejorar las actividades agrícolas de algunas zonas en el lugar de estudio, por lo que el objetivo de esta investigación fue determinar la efectividad del sistema de bombeo con ariete hidráulico en la zona rural de la provincia de San Pablo-Cajamarca.

\section{MATERIALES Y MÉTODOS}

La investigación presentó la siguiente secuencia de actividades para su ejecución: 
Etapa de campo: se trató de la localización geopolítica de la zona, de los recursos disponibles, determinación de la altura disponible y de descarga, ubicación de la toma de agua y tanque de captación, construcción e instalación del sistema de bombeo con ariete hidráulico, determinación de la ubicación del tanque de descarga.

Para poder garantizar que las pruebas tuviesen la suficiente fiabilidad, se aseguró que el tanque de alimentación (o sistema fluyente) estuviese libre de impurezas y que proporcionara una cantidad de agua de alimentación constante, se establecieron las características de la prueba a realizar, regulando los accesorios de la válvula de impulso, abriendo la válvula de compuerta, para permitir el paso de agua a la bomba, el ariete comenzará su funcionamiento cerrando y abriendo la válvula, caso contrario, se produce el golpe de ariete, para inducir la abertura de las válvulas manualmente hasta que se establezca el golpe y seguir el paso anterior; una vez que se estabilizó el funcionamiento de la bomba, se tomaron los datos de altura de elevación (h), la altura de trabajo $(H)$, y la longitud de la tubería de alimentación (L) (Cengel \& Cimbala, 2006; Jara et al., 2011; Tuquinga \& Paredes, 2013).

Etapa de gabinete: se aplicó el cuestionario para medir la satisfacción del poblador rural de uso y aplicación del sistema de bombeo con ariete hidráulico; se realizó el análisis económico, donde se determinaron los indicadores económicos, para validar su viabilidad económica.

Para determinar la satisfacción del poblador rural, se aplicó un cuestionario basándose en cinco factores. Cada pregunta, según la respuesta dada, se suma, donde la puntuación de los ítems determinará su grado de satisfacción, el cual comprendería las valoraciones de las personas en relación al uso y aplicación de los sistemas de bombeo con ariete hidráulico en sus actividades cotidianas.

Para el análisis de datos, se elaboraron los diagramas de parámetros de operación del sistema de bombeo con ariete hidráulico, parauna mejor interpretación de los resultados obtenidos.
Con los materiales utilizados en la construcción de la bomba de ariete, se obtuvieron los costos de fabricación y los gastos que se emplearon para su instalación y operación, lo cual determinaron los indicadores económicos y viabilidad económica según la realidad del poblador y el tiempo de vida útil del sistema de bombeo con ariete hidráulico (Quiroga et al., 2013).

\section{RESULTADOS \\ Efecto de la altura de elevación (h)}

En la Figura 1, se observa que la cantidad de agua de alimentación es la misma para las tres configuraciones. Esto sucede porque la presión en la tubería de elevación, no influye para nada, por lo que la cantidad de agua de alimentación por ciclo $\left(\mathrm{Q}_{\text {ciclo }}\right)$ deberá ser la misma para cualquier altura de elevación (h), y como el tiempo de ciclo es prácticamente el mismo, el agua gastada por minuto $(\mathrm{Q})$ apenas varía. 


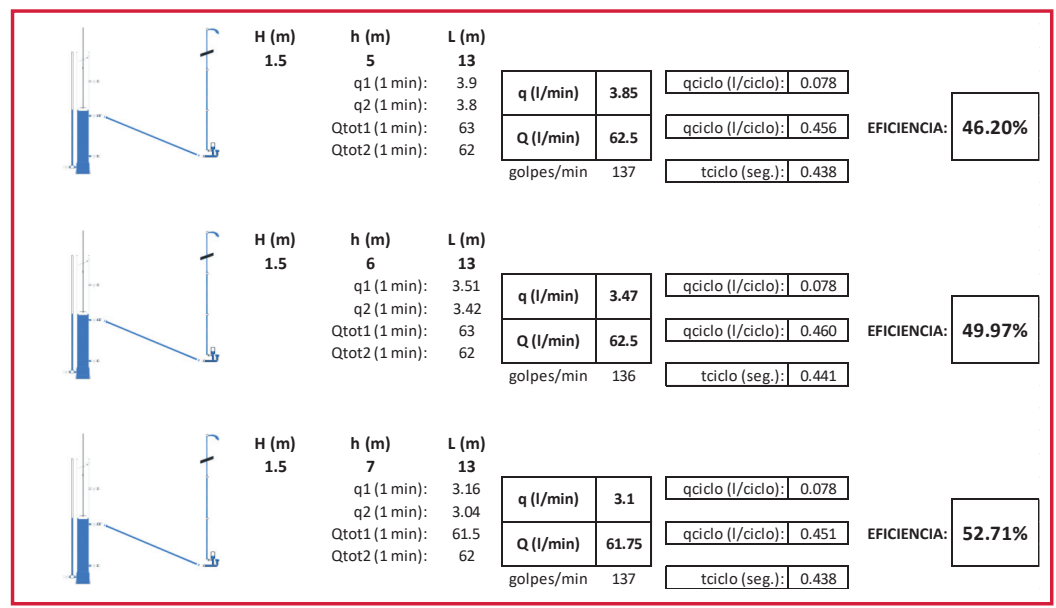

Figura 1. Efecto de la altura de salida (descarga) h en la bomba de ariete

En la Figura 2, se muestra la tendencia de la curva de la eficiencia de la bomba de ariete, la cual refleja el comportamiento de los parámetros evaluados (Tabla 1), como (Q, q y $\eta$ ). Una forma de poder realizar una comparación entre la parte teórica y práctica es, a través de un modelo teórico elaborado en MathCad, dada por Acitores (2012). Para ello se asignaron diferentes valores teóricos a h, para observar cómo se comportaban las variables medidas según el modelo; por lo que se comporta de manera similar a la realidad en cuanto a rendimiento y caudales por minuto.

Tabla 1

Comportamiento de los parámetros evaluados por efecto de la altura de elevación

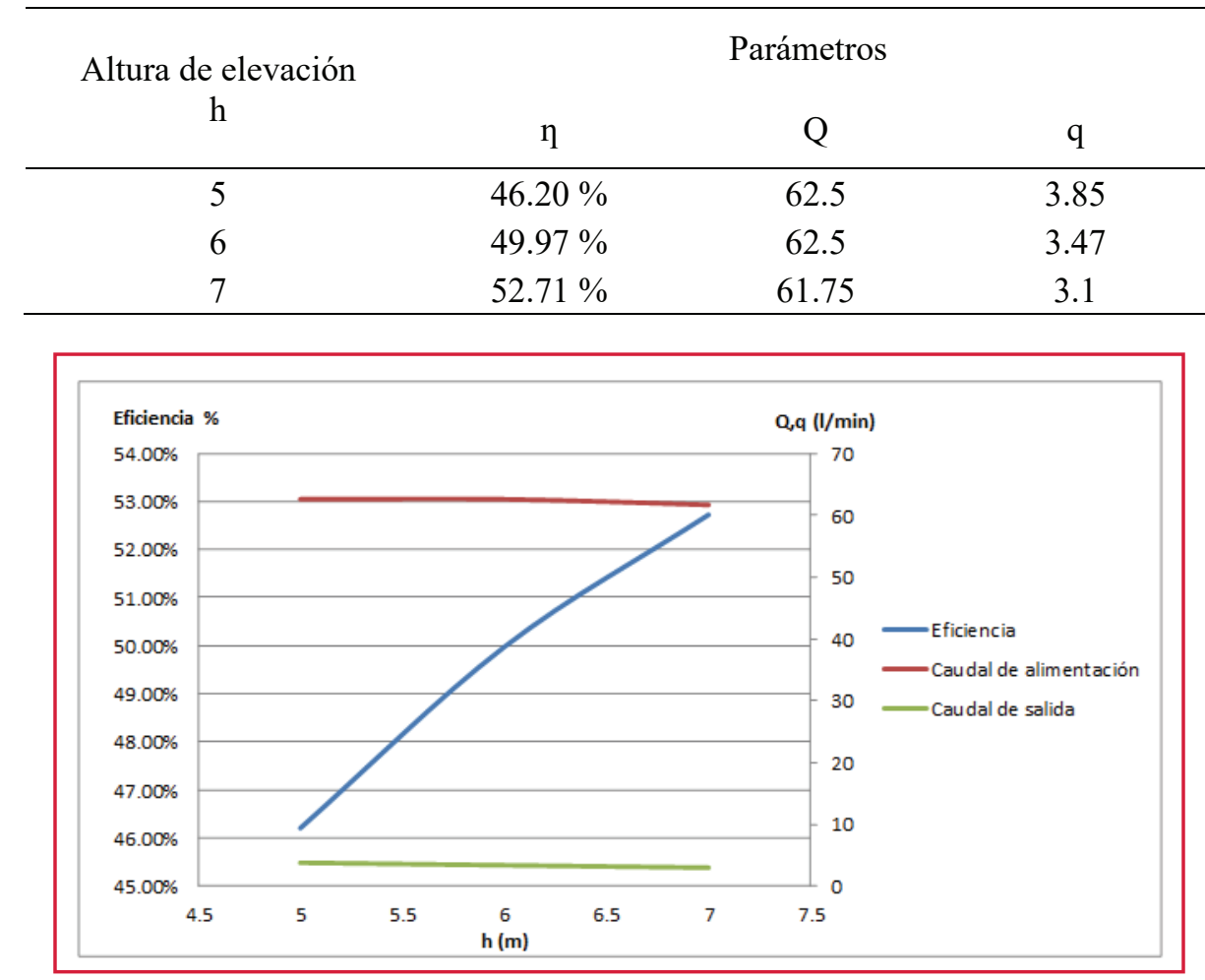

Figura 2. Comportamiento de la bomba de ariete en función de la altura de elevación 


\section{Efecto de la altura de alimentación $(\mathrm{H})$}

Los resultados de las Figuras 3 y 4, evidencian que el tiempo de ciclo disminuye a medida que aumenta la altura de alimentación (Tabla 2), ya que el fluido se acelera mucho más rápidamente; al haber más presión de alimentación, alcanza más rápido la velocidad necesaria para cerrar la válvula de choque y comenzar un nuevo ciclo. Un comportamiento curioso es el del caudal gastado $(\mathrm{Q})$, que parece que no se ve afectado por la altura de alimentación.

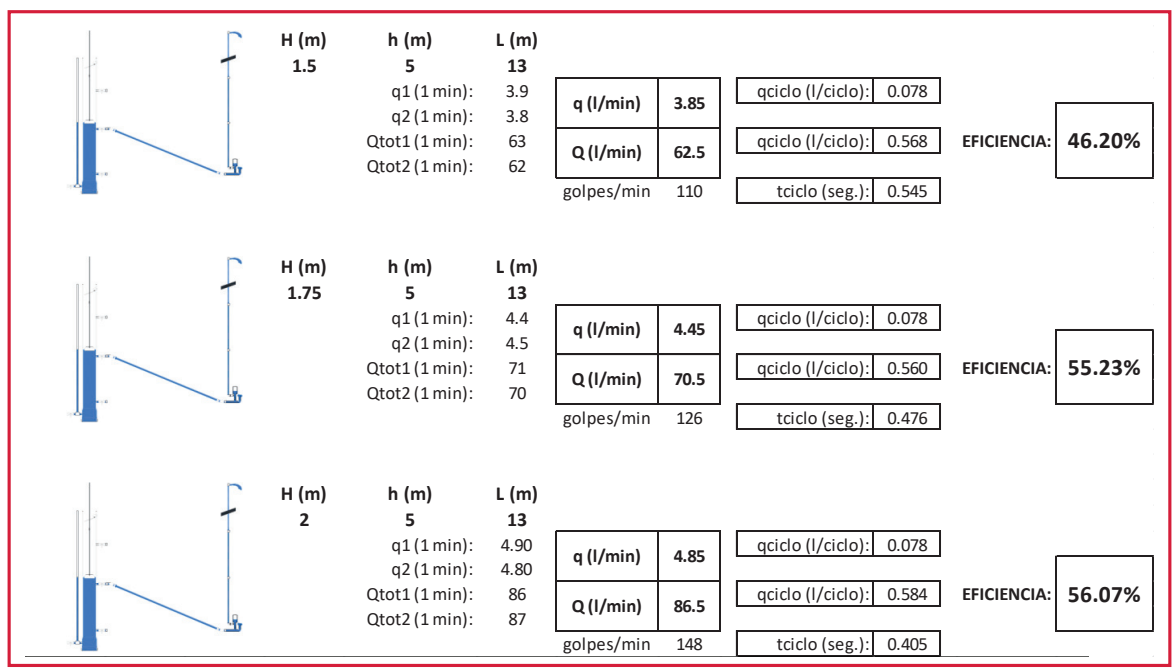

Figura 3. Efecto de la altura de alimentación $\mathrm{H}$ en la bomba de ariete

Tabla 2

Comportamiento de los parámetros evaluados por efecto de la altura de alimentación

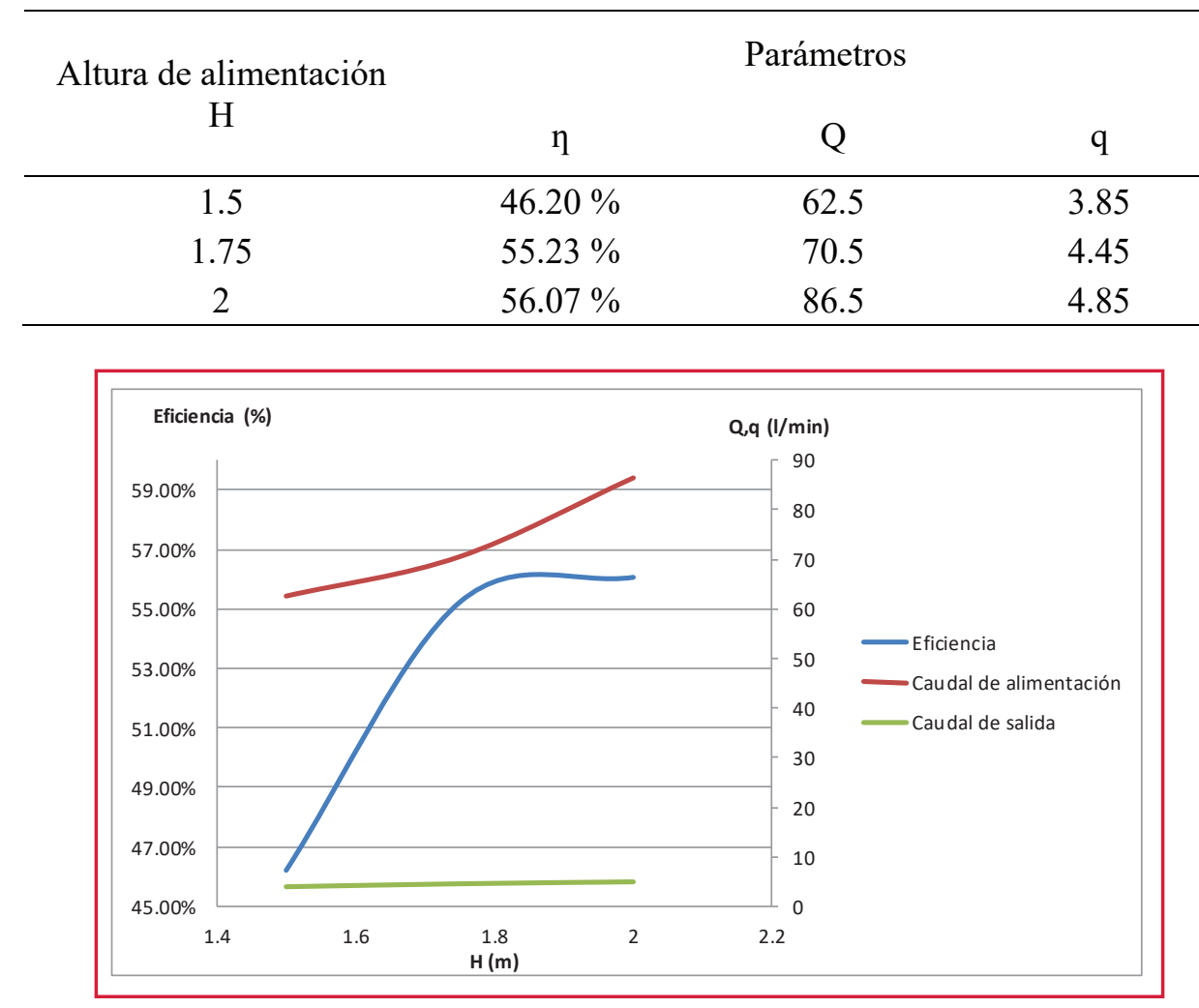

Figura 4. Comportamiento de la bomba de ariete en función de la altura de alimentación 
En la Figura 4, los valores experimentales con los obtenidos según las ecuaciones del modelo teórico mencionado anteriormente (Acitores, 2012), permite comparar el rendimiento teórico con los datos obtenidos.
Efecto de la longitud de la tubería de alimentación (L)

En este caso, se mantiene constante la altura de elevación ( $\mathrm{h}=5 \mathrm{~m}$ ), la altura de alimentación ( $\mathrm{H}$ $=1.5 \mathrm{~m}$ ), y se prueba con diferentes longitudes de tubería (Tabla 3), así se puede lograr con la combinación de tramos de tubería que se tiene, siempre manteniendo una inclinación de 25을 (Figura 5).

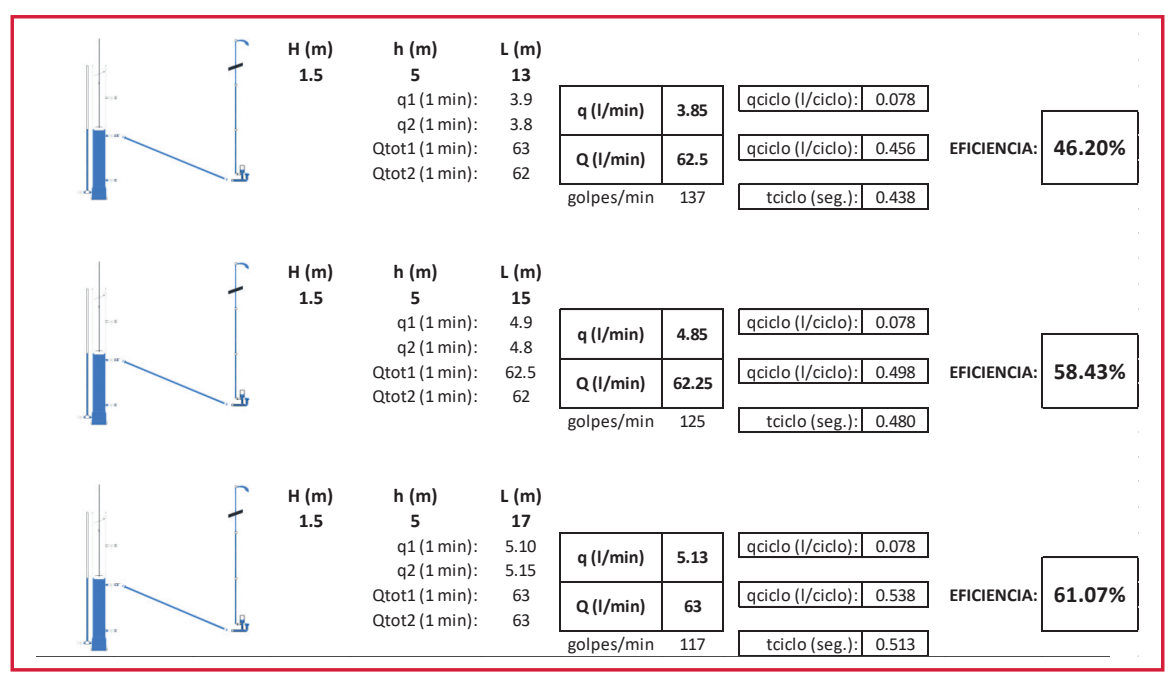

Figura 5. Efecto de la tubería de alimentación ( $\mathrm{L}$ ) en la bomba de ariete

Tabla 3

Comportamiento de los parámetros evaluados por efecto de la longitud de la tubería de alimentación

\begin{tabular}{cccc}
$\begin{array}{c}\text { Tuberia de Alimentación } \\
\text { L }\end{array}$ & $\eta$ & $\mathrm{Q}$ & $\mathrm{q}$ \\
\hline 13 & $46.20 \%$ & 62.5 & 3.85 \\
15 & $58.43 \%$ & 62.25 & 4.85 \\
17 & $61.07 \%$ & 63 & 5.13 \\
\hline
\end{tabular}




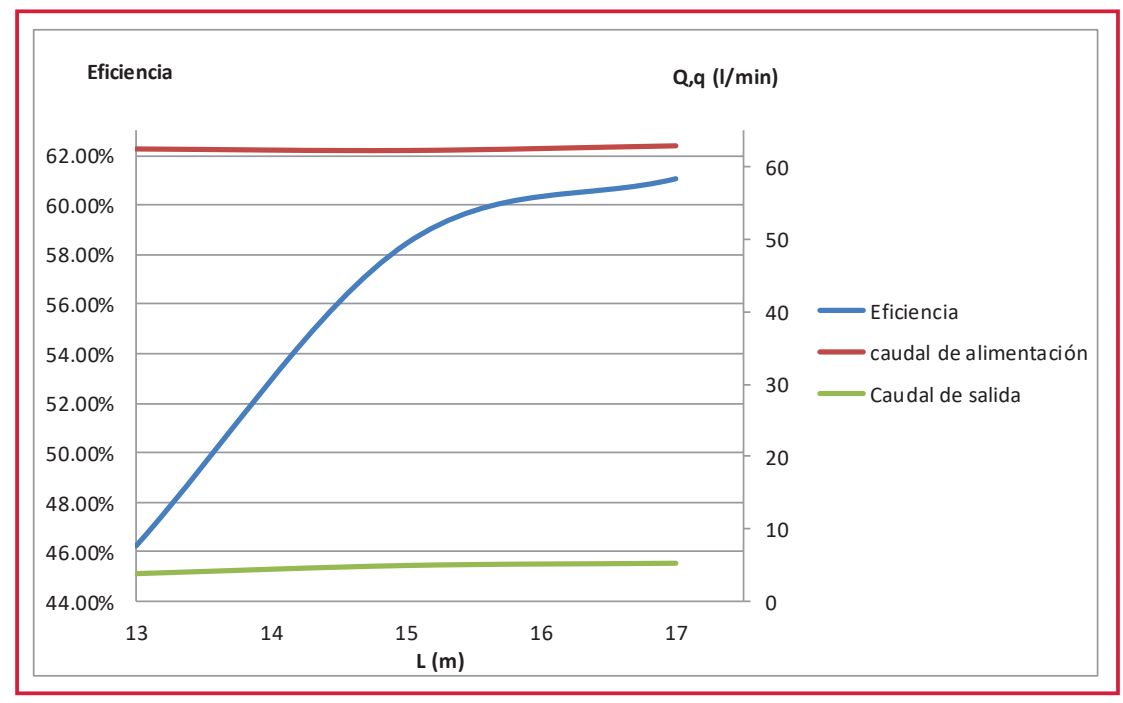

Figura 6. Comportamiento de la bomba de ariete en función de la tubería de alimentación

Análisis técnico económico de la bomba de ariete vs un motor de combustión (motobomba). En la Comunidad de Suro Antivo, Tumbadén, San Pablo, se realizó la evaluación en seis familias (de cuatro integrantes aprox/familia).
El consumo de agua por persona: es aprox. 25-30 l/min; el total de cabezas de ganado de estas familias, fue 26 y, el consumo de agua por animal (vaca) aprox. 25-35 l/min.

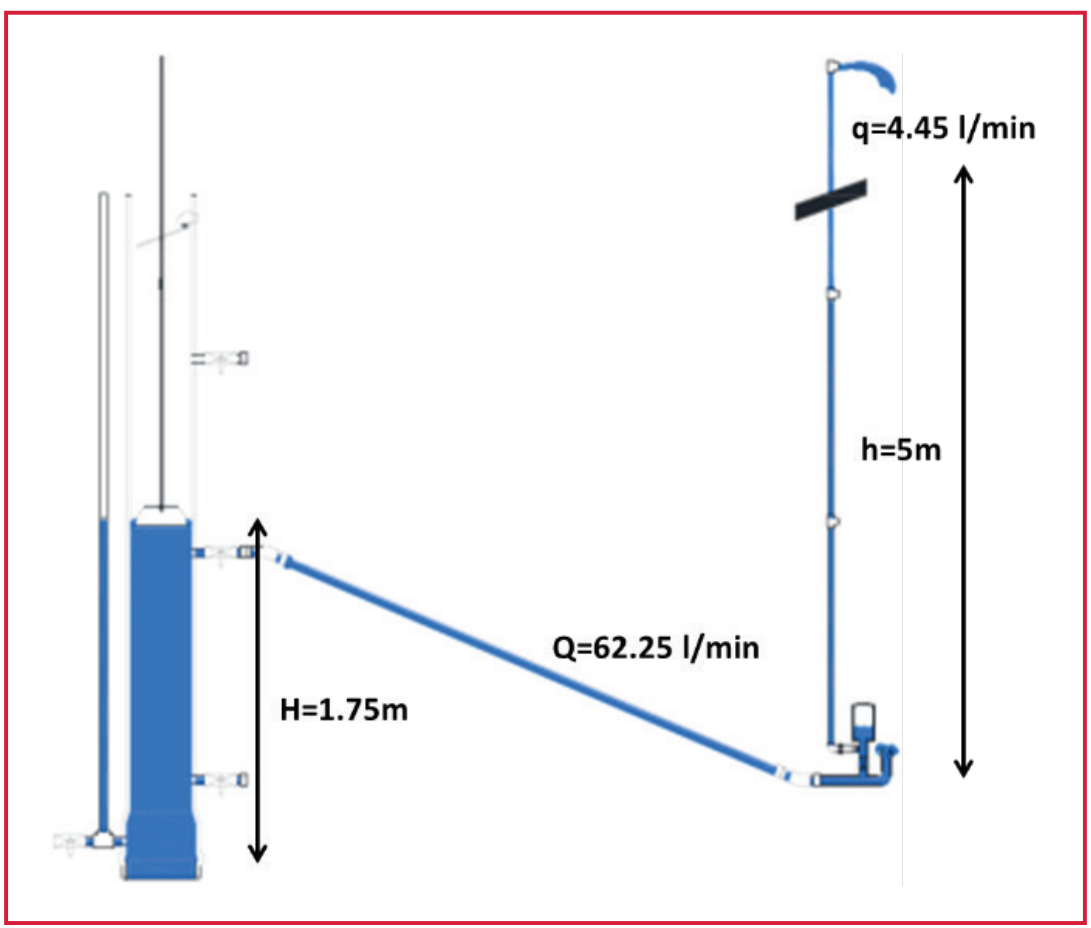

Figura 7. Configuración óptima del sistema de bombeo individual 
La demanda total de agua para el ganado 0.632 l/min, para las pasturas $6.94 \mathrm{l} / \mathrm{min}^{\star}$ hectárea y doméstica $0.5 \mathrm{l} / \mathrm{min}$, por lo que la demanda total es de $8.072 \mathrm{l} / \mathrm{min}$ de agua; con esta demanda hídrica de 8.072 l/min, la mejor opción es el abastecimiento de agua por medio de dos bombas de ariete hidráulico conectados en paralelo, para incrementar el caudal en con cuerpo de bomba 1", según la Figura 7, la cual muestra la capacidad que tiene una bomba de ariete hidráulico para elevar $4.45 \mathrm{l} / \mathrm{min}$ de agua; por lo que es necesario conectar dos sistemas conectados en paralelo y de esta manera abastecer las diferentes demandas de la zona en estudio con un total de $8.9 \mathrm{l} / \mathrm{min}$.

Según Mosquera et al. (2010)., un motor de combustión interna que utiliza gasolina como carburante, emite $2.28 \mathrm{Kg}$ de $\mathrm{CO}_{2}$ por cada litro de gasolina que combustiona; y considerando los parámetros de operación de la motobomba; la cantidad de emisión de $\mathrm{CO}_{2}$ sería de:

$$
\begin{gathered}
\text { Cantidad de } \mathrm{CO}_{2}=\frac{1.75 \mathrm{lt}}{\text { día }} * \frac{2.28 \mathrm{Kg} \mathrm{de} \mathrm{CO}_{2}}{l t} \\
\text { Cantidad de } \mathrm{CO}_{2}=4.0 \frac{\mathrm{Kg} \mathrm{de} \mathrm{CO}_{2}}{\text { día }} \\
\text { Cantidad de } \mathrm{CO}_{2}=120.0 \frac{\mathrm{Kg} \mathrm{de} \mathrm{CO}_{2}}{\mathrm{mes}}
\end{gathered}
$$

Siendo, ésta la cantidad de $\mathrm{CO}_{2}$, que se emitiría a la atmosfera, si se utilizara una motobomba para las consideraciones de operación expuestas anteriormente. Además, se consideraría que la máquina como tal, no puede estar parada por lo que probablemente se usaría en otros lugares emitiendo mayor cantidad de $\mathrm{CO}_{2}$ al ambiente; mientras que con el sistema de bombeo con ariete hidráulico no habría emisiones. Económicamente es más rentable el sistema de bomba de ariete, ya que la alternativa seleccionada, para dos proyectos de mejora, se elige el que cuesta menos; además, teniendo en cuenta que este requerimiento y la situación actual de la comunidad, es necesario utilizar el sistema de bombeo con ariete hidráulico.

\section{Análisis desde el punto de vista social}

De acuerdo a la Figura 8, se considera que el nivel de satisfacción que proporciona el uso y aplicación de esta tecnología es positivo; donde, el sistema de bombeo con ariete hidráulico es una tecnología positiva desde el punto de vista técnico, económico y ambiental. Cabe mencionar que, la encuesta fue aplicada a 10 pobladores directos de la zona en estudio; tal cantidad se debió a aquellas personas que estuvieron prestas a participar en el estudio, ya que ciertos pobladores, tienen la consigna que cualquiera investigación que se realiza en la zona va con intereses negativos.
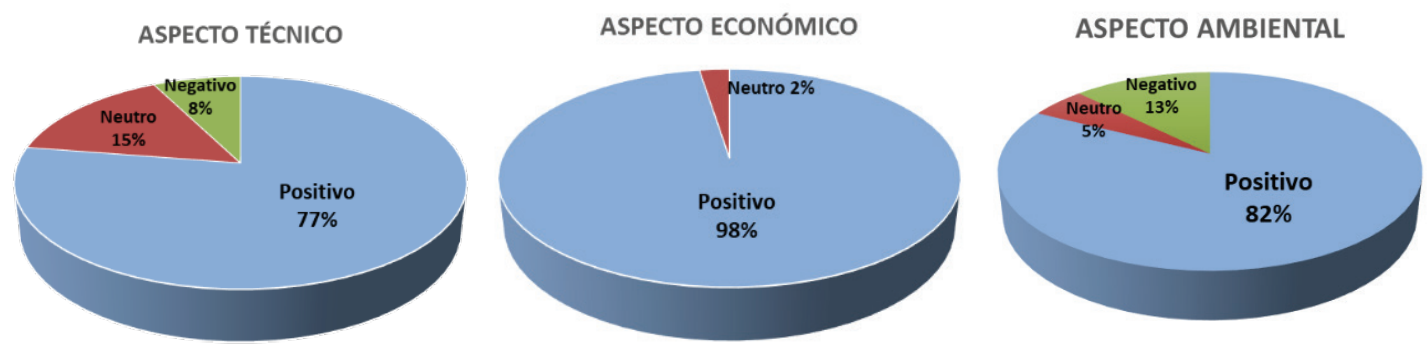

Figura 8. Percepción técnica, económica y ambiental del poblador 
Según lo mencionado anteriormente, desde el punto de vista social tendría una repercusión muy importante, ya que es una tecnología que no contamina, es muy económica y que permite tener acceso a uno de los recursos, si no, el más importantes del planeta; el agua. Este a su vez, permite mejorar las condiciones de salud del poblador, porque no solo el agua se utilizaría para actividades de producción si también domésticas.

\section{DISCUSIÓN}

De los resultados obtenidos, el parámetro que varía, como es de esperar, es el caudal de agua elevada (q), que disminuye a medida que la altura de elevación es mayor. Sin embargo, el rendimiento es mayor cuanto más alta la altura de elevación, por lo que es más eficiente elevar menos agua a más altura. Esto coincide con Acitores (2012), donde, según las características de esta tecnología, logran alcanzar valores de rendimiento entre $29.6 \%$ y $42.1 \%$, considerando en promedio valores de $75 \mathrm{gpm}$ de la válvula de derrame, mientras que en este trabajo se alcanzaron cifras de $125 \mathrm{gpm}$, obteniendo rendimientos alrededor del $51 \%$, valores sobre el promedio de esta tecnología. Siendo más específico, en el caso del modelo (Acitores, 2012), se logra un rendimiento máximo del $37 \%$, para una altura de alimentación de $1,25 \mathrm{~m}$, rendimiento que disminuye a medida que se aleja de este valor tanto en un sentido como en otro. El rendimiento experimental resultó del 56.07 $\%$, tomando como altura de alimentación de 2 metros (a diferencia de 1 metro según el modelo); lo que le permite alcanzar valores de eficiencia mayor; y lo mismo ocurre con los parámetros de evaluación para cada simulación.

Tal como lo señala la Agencia Internacional de Cooperación Japonesa JICA (2012), el acceso al riego de bajo costo es un factor que puede tener un impacto económico y social positivo muy amplio e importante en la vida de las familias de pequeños productores tradicionales. Por su simplicidad y bajo costo, la bomba de ariete se adapta a las condiciones de los pequeños productores de escasos recursos económicos y técnicos; también referido por Tuquinga \& Paredes (2013).

- Con este proyecto, la inversión total del sistema asciende a S/. 528.00 nuevos soles, incluyendo accesorios, lo que permite ser accesible para las personas del lugar o en zonas de similares características; teniendo una aceptación no solo por correcto funcionamiento si no por la sencillez en la construcción y operación; además, de ser amigable con el medio ambiente. Por último, el nivel de satisfacción del poblador rural de la zona en estudio es positivo, desde los aspectos, técnico, económico y ambiental, para con la tecnología del sistema de bombeo con ariete hidráulico.

\section{CONCLUSIONES}

- La efectividad del sistema de bombeo con ariete hidráulico en la zona rural de la Provincia de San Pablo - Cajamarca es la más óptima bajo las circunstancias de trabajo analizadas, ya que la eficiencia del equipo es de $55.23 \%$ y es más económica desde su inversión hasta sus costos de operación y mantenimiento.

- La demanda total del recurso hídrico en la parte superior de la zona en estudio, es de 8.072 l/min; lo cual considera, demanda por ganado $(0.632 \mathrm{l} / \mathrm{min})$, demanda por riego de pasturas $(6.94 \mathrm{l} / \mathrm{min})$ y demanda doméstica (0.5 l/min), mientras que la oferta la proporciona un canal la cual alimenta la zona baja del lugar, proporcionando para ello de 15 a $25 \mathrm{l} / \mathrm{s}$ de agua.

- Los parámetros de diseño y operación del sistema de bombeo con ariete hidráulico son: cuerpo de la bomba 1", altura de alimentación $H=1.75 \mathrm{~m}$, altura de descarga $\mathrm{h}=5 \mathrm{~m}$, caudal de alimentación $\mathrm{Q}=62.25 \mathrm{l} / \mathrm{min}$ y el caudal de descarga $\mathrm{q}=4.45 \mathrm{l} / \mathrm{min}$; como el sistema será una instalación en paralelo; esta proporciona 8.9 L/min para cubrir la demanda. Cabe mencionar que esta configuración tiene una eficiencia total del sistema de $55.23 \%$ siendo un valor número muy aceptable para este tipo de tecnología.

- Desde el punto de vista económico es más rentable ya que al comparar con una 
motobomba, el VAC de la bomba de ariete hidráulico es de S/. 1521.29 mientras que de la motobomba es de S/.3157.73, siendo la primera alternativa (bomba de ariete hidráulico) la de menor costo.

\section{AGRADECIMIENTO}

El autor agradece a la institución Soluciones Prácticas - ITDG Sede Cajamarca por su apoyo y colaboración en las diferentes actividades prácticas realizadas en sus instalaciones y visitas de campo en la zona de estudio.

\section{REFERENCIAS BIBLIOGRÁFICAS}

Acitores, M. (2012). Estudio teórico y experimental de la bomba de ariete. [Tesis de grado]. Universidad Carlos III de Madrid. Disponible en: https://core.ac.uk/ download/pdf/30046069.pdf

Arapa, J. (2016). Evaluación del rendimiento del ariete hidráulico bah-1.1/2 variando la longitud de la tubería de alimentación y condiciones de operación de la válvula de impulso. Anales Científicos, 77(2),155165 DOl: http://dx.doi.org/10.21704/ ac.v77i2.485

Cengel, Y. \& Cimbala. J. (2006). Mecánica de Fluidos, Fundamentos y Aplicaciones. 1a edición. México. McGraw-Hill.

Mayorga, A. \& Galarza, R. (2013). Estudio de Factores Hidráulicos en una Bomba de Ariete y su efecto sobre la eficiencia [Tesis de pregrado]. Universidad Técnica de Ambato Ecuador. Disponible en: https://repositorio.uta.edu.ec/jspui/ handle/123456789/3714

Jara, N., Campoverde. E. \& Pizarro, D. (2011). Emplazamiento, implementación, pruebas de funcionamiento y propuestas de mejora de los sistemas de bombeo mediante rueda hidráulica y ariete multipulsor para el abastecimiento de agua para irrigación en la localidad del campus UPS - Juan Lunardi - Yumacay - Paute - Azuay". Ingenius. Revista de Ciencia y Tecnología, 6, 51-62. Disponible en: $\quad$ https://www.redalyc.org/articulo. oa? id $=505554810007$

JICA (2012). Guía Agropecuaria: Estudio de Promoción y Difusión de Buenas Prácticas "Bomba de Ariete" del Proyecto Tawan Ingnika. Disponible en: https://docplayer. es/10210717-Estudio-de-promocion-ydifusion-de-buenas-practicas-bomba-deariete-del-proyecto-tawan-ingnika.html.

Mosquera, J., Fernández, S. y Mosquera J.C. (2010). Análisis de emisiones de CO2 para diferentes combustibles en la población de taxis en Pereira y Dosquebradas. Scientia et Technica, 2(45), 141 - 146. DOl: https://doi. org/10.22517/23447214.385

Nwosu, C. \& Madueme, T. (2013). Recycled Micro Hydropower Generation Using Hydraulic Ram Pump (Hydram). IMPACT: IJRET, 1(3), 1-10. Disponible en: http:// paper.researchbib.com/view/issn/2321$8843 / 1 / 3$

Ortega, O. \& Gonzáles, C. (2013). Construcción, caracterización hidráulica y estudio de aplicación de una bomba de ariete para el riego de una finca agrícola [Tesis de Grado]. Universidad de Zaragoza. Disponible en: https://zaguan.unizar.es/ record/10487? ln=es\#.

Quiroga, M., Sierra, G. y Biancha, G. (2013). Análisis del ariete hidráulico para diferentes configuraciones. Revista UIS Ingenierías, 12(2), 29-34. Disponible en: https://www. redalyc.org/pdf/5537/553756870003.pdf

Tuquinga, R. \& Paredes, M. (2013). Diseño e instalación de un sistema de bombeo mediante ariete hidráulico en la comunidad de Airón Cebadas [Tesis de pregrado]. Escuela Superior Politécnica Chimborazo, Ecuador. Disponible en: http://dspace. espoch.edu.ec/handle/123456789/2403 